\title{
Improvement of rosacea by injection of platelet-rich plasma
}

\author{
Uekyoung Hwang, MD, MPh \\ Yeline Clinic, Seongnam, Rep. of Korea
}

\begin{abstract}
Rosacea is a chronic facial inflammatory disease characterized by erythema, papules, telangiectasia, and recurrent flushing. Many studies have documented the crucial role that an immature immune system plays in the progression of rosacea. Various studies have illustrated the potential effectiveness of platelet-rich plasma (PRP) on the inhibition of inflammation. Although rosacea is a disease caused by inflammation accompanied by an immature immune system, there is no research explaining the effects of PRP on rosacea through the regulation of immunity and inflammation. The PRP was prepared using the SmartPReP®2 APC+ ${ }^{\mathrm{TM}}$ in three participants who provided informed consent. The centrifuged PRP (total 4-5 ml) was then injected into the facial dermal area using a 26-gauge $5-\mathrm{cm}$ cannula. In all three cases, the erythema in the cheek and chin area was severe, and telangiectasia was seen in the cheek preoperatively. Postoperatively, erythema and telangiectasia improved significantly during weeks 4 and 12 . We believe this is the first article to document the potential therapeutic effects of PRP enriched with $\mathrm{CD}_{3} 4^{+}$stem cells in the peripheral blood for the treatment of rosacea. In conclusion, PRP enriched with $\mathrm{CD}_{3} 4^{+}$stem cells in the peripheral blood may have a potential additive therapeutic effect on rosacea via an immunomodulation effect. Long-term prospective follow-up studies are warranted to confirm the beneficial effect of PRP enriched with $\mathrm{CD}_{3} 4^{+}$stem cells in the peripheral blood for the treatment of rosacea.
\end{abstract}

Keywords: CD34; dermatitis; platelet-rich plasma; rosacea

\section{Introduction}

Rosacea is a chronic facial inflammatory disease that is characterized by erythema, papules, telangiectasia, and recurrent flushing [1]. Patients with rosacea do not have a protective layer of skin. This causes excessive transdermal water loss which makes the skin dry and prone to peeling and results in a burning or stinging face. The etiology of rosacea has not been identified; however, abnormalities of the immune, vascular, and nervous systems as well as microorganisms such as Demodex folliculorum may be involved in the pathogenesis of rosacea [1]. Numerous studies have shown that an immature immune system plays a crucial role in the progression of rosacea. It has been reported that the levels of toll-like receptor 2 (TLR2), cathelicidin antimicrobial peptide (CAMP), and kallikrein 5 (KLK5) are increased in patients with rosacea [2]. Moreover, the significant peptide CAMP LL-37 produces rosacea-like skin lesions. In addition to immunological abnormalities, vascular and nervous dysfunctions play a crucial role in the pathogenesis of rosacea. Histopathological findings of rosacea are blood vessel dilatation, angiogenesis, and an increase in nerve fibers. Treatment modalities for rosacea can be divided into two categories which control inflammation and inhibition of vessel dilatation [3]. In this regard, the Food and Drug Administration (FDA) approved

Received December 4, 2019; Revised December 23, 2019; Accepted December 28, 2019

Corresponding author: Uekyoung Hwang

E-mail: yjukhome@daum.net

This is an Open Access article distributed under the terms of the Creative Commons Attribution Non-Commercial License (http://creativecommons.org/licenses/by-nc/4.0), which permits unrestricted non-commercial use, distribution, and reproduction in any medium, provided the original work is properly cited.

Copyright ( 2019 Korean Society of Korean Cosmetic Surgery and Medicine (KSKCS \& KCCS). 
doxycycline as an inflammatory regulator and bromonidine as a vasoconstrictor. More recently, platelet-rich plasma (PRP) has been used to promote wound healing in the hard and soft tissues of various medical conditions and in stem cell-based tissue engineering [4]. Activation of platelets trigger degranulation and release of growth factors that influence wound healing, tissue regeneration, angiogenesis, and stem cell activation. PRP secretes several types of growth factors such as platelet-derived growth factor (PDGF), epithelial growth factor (EGF), vascular endothelial growth factor (VEGF), endothelial cell growth factor (ECGF), fibroblast growth factor (FGF), transforming growth factor beta (TGF- $\beta$ ), and insulin-like growth factor (IGF) [5]. In addition to the direct effect of PRP on cell regeneration, differentiation, and angiogenesis, PRP also affects tissue regeneration through the chemotactic recruitment of stem cells and local control of inflammatory conditions [5].

Many studies have reported the potential ability of PRP to inhibit inflammation. The activated PRP demonstrates high levels of hepatocyte growth factor (HGF), interleukin 4, tumor necrosis factor- $\alpha$, and interleukin 1 . In a tendon cell model, PRP induces the expression of VEGF, regulated on activation, normal T-cell expressed and secreted chemokine (RANTES), and HGF and decreases pro-inflammatory cytokines such as interleukin
6, interleukin 8, and monocyte chemoattractant protein 1 [5]. Although rosacea is a disease caused by inflammation accompanied by an immature immune system, there is no research that illustrates the effect of PRP on rosacea through the regulation of immunity and inflammation. In this case study, the SmartPReP ${ }^{\circledR} 2$ APC $+{ }^{\mathrm{TM}}$ (Harvest Technologies Corp., Plymount, MA, USA), approved by FDA in the United States, was used to prepare the PRP. This study aimed to investigate whether PRP extracted through the SmartPReP ${ }^{\circledR}$ system reduces the redness of rosacea by regulation of immunity and inflammation.

\section{Case report}

The PRP was prepared using the SmartPReP ${ }^{\circledR} 2 \mathrm{APC}^{\mathrm{TM}}{ }^{\mathrm{T}}$ (Harvest technologies Corp.) from three participants who provided informed consent. A total of $30 \mathrm{ml}$ of blood obtained from each participant was transferred to a tube containing $5 \mathrm{ml}$ of $4 \%$ sodium citrate solution (Nothrom; Daehwa Pharmaceutical., Hwaseong, Korea). The condition for extracting the PRP by centrifugation was 4 minutes at 2,500 RPM and the concentration was 10 minutes at 2,300 RPM. The participant's face was cleansed with a betadine swab, and local anesthesia with $2 \%$ lidocaine with 1:100,000 epinephrine was applied. The centri-
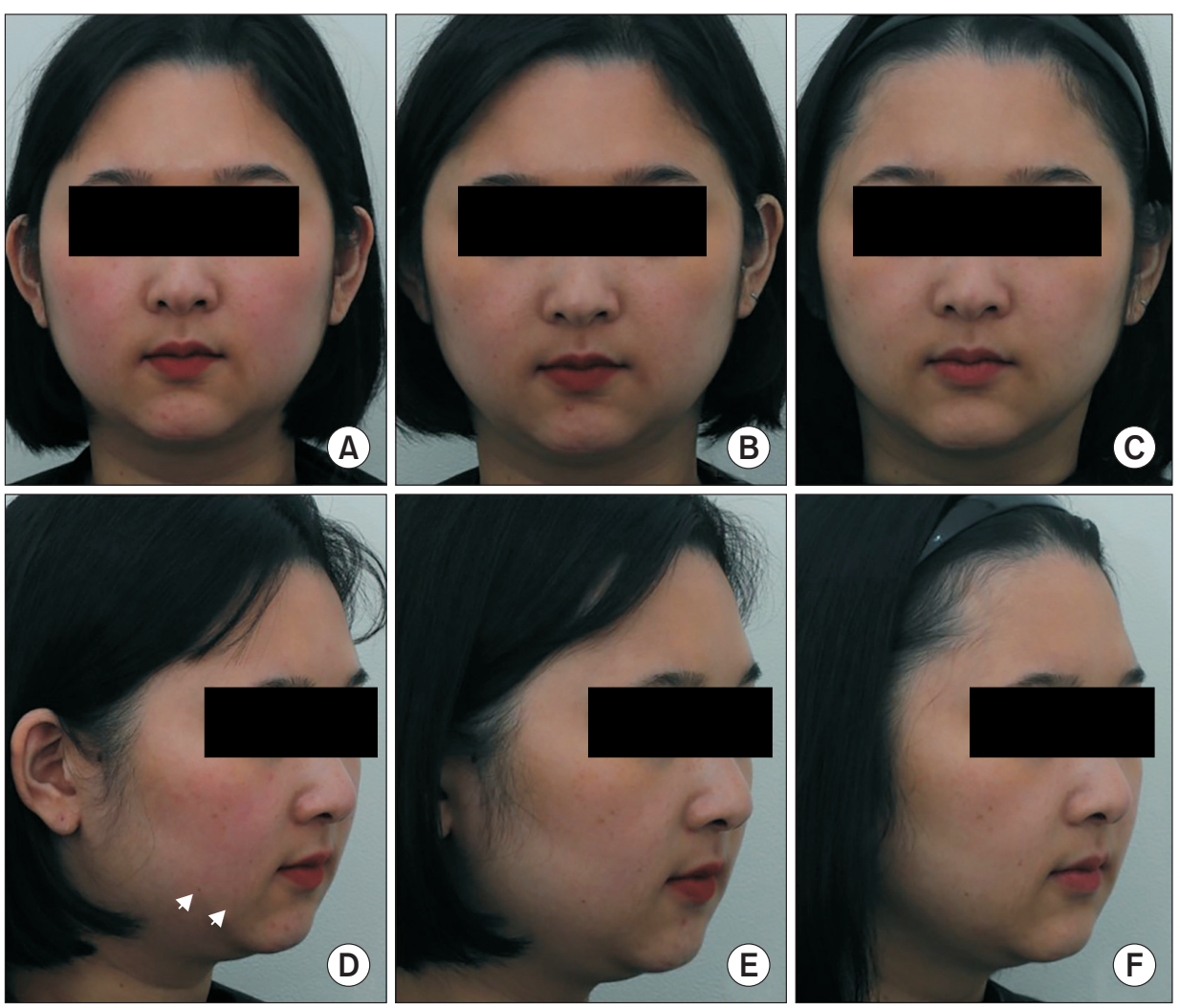

Fig. 1. A 28-year old woman had atopic dermatitis from an early age with severe itching. (A, D) Before platelet-rich plasma (PRP), erythema in cheek and chin area was severe, and telangiectasia was seen in lateral cheek. (C, F) Postoperatively, erythema and telangiectasia improved significantly. White arrow: telangiectasia. 
fuged PRP (total 4-5 ml) was injected into the facial dermal area using a 26-gauge 5 -cm cannula. Following the PRP procedure, the three patients were followed for 1 week, 1 month, 2 months, and 3 months. We evaluated the improvement of the rosacea, and the skin texture and tone.

\section{Case 1}

This patient had atopic dermatitis from an early age with severe itching during the spring and autumn seasons with dryness and hot flushes during the winter. Preoperatively, the erythema in the cheek and chin area was severe, and telangiectasia was seen in the lateral cheek area (Fig. 1A, D). Postoperatively, the erythema and telangiectasia improved significantly during weeks 4 and 12, and the patient's sense of itching and dryness also improved over baseline (Fig. 1C, F).

\section{Case 2}

This participant had severe recurrent facial flushing with a sense of body temperature fluctuations and continuous folliculitis in the chin area. Preoperatively, erythema and telangiectasia were observed across the cheek and chin, especially in the chin area folliculitis (Fig. 2A, D). Postoperatively, the recurrent facial flushing with temperature change significantly improved and the telangiectasia of the lower lateral cheek area also improved (Fig. 2C, F). Specifically, the patient was satisfied with the improvement of folliculitis in the chin area.

\section{Case 3}

This participant had very fragile skin from exposure to the sun and suffered from multiple senile keratosis and erythema surrounding the cheek area after playing golf in the recent years.

Preoperatively, there was severe erythema and multiple senile keratosis on the cheek. Telangiectasia was observed around the nasal alar area (Fig. 3A, D). Postoperatively, the erythema and senile keratosis were much improved, and the telangiectasia around the nasal alar area also improved. Further, the irregular skin texture due to sebum and moisture imbalance was stabilized (Fig. 3C, F).

\section{Discussion}

Rosacea is a chronic inflammatory skin disease affecting approximately $3 \%$ of world's population and is characterized by erythema, papules, telangiectasia, and recurrent flushing. It is
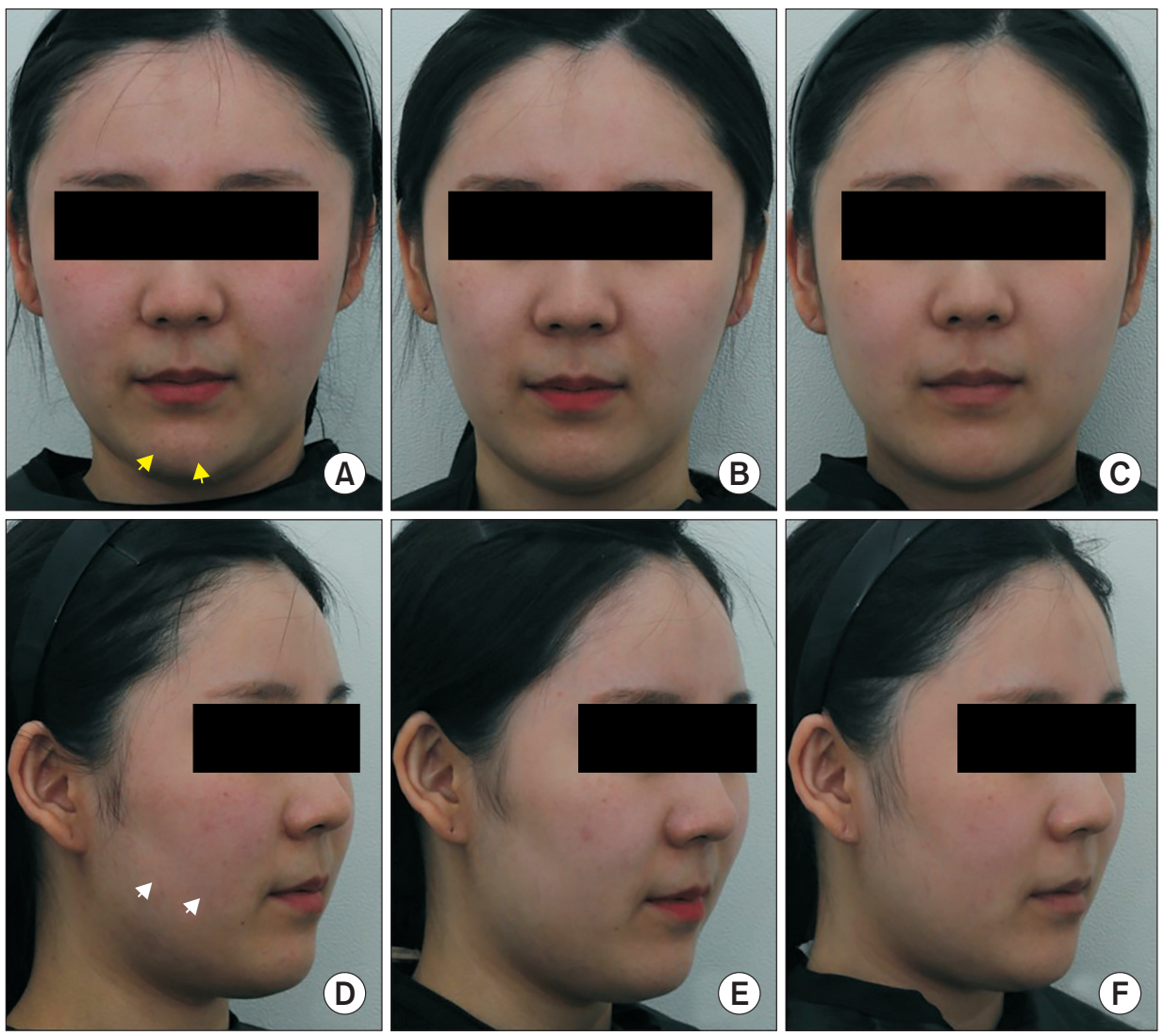

Fig. 2. A 30-year old woman had severe recurrent facial flushing with a sense of body temperature fluctuation. (A, D) Before platelet-rich plasma (PRP) procedure erythema and telangiectasia are observed across the cheek and chin, especially in the chin area folliculitis. (C, F) Postoperatively, facial recurrent flushing and lower lateral cheek area telangiectasia improved. White arrow: telangiectasia, yellow arrow: chin area folliculitis. 

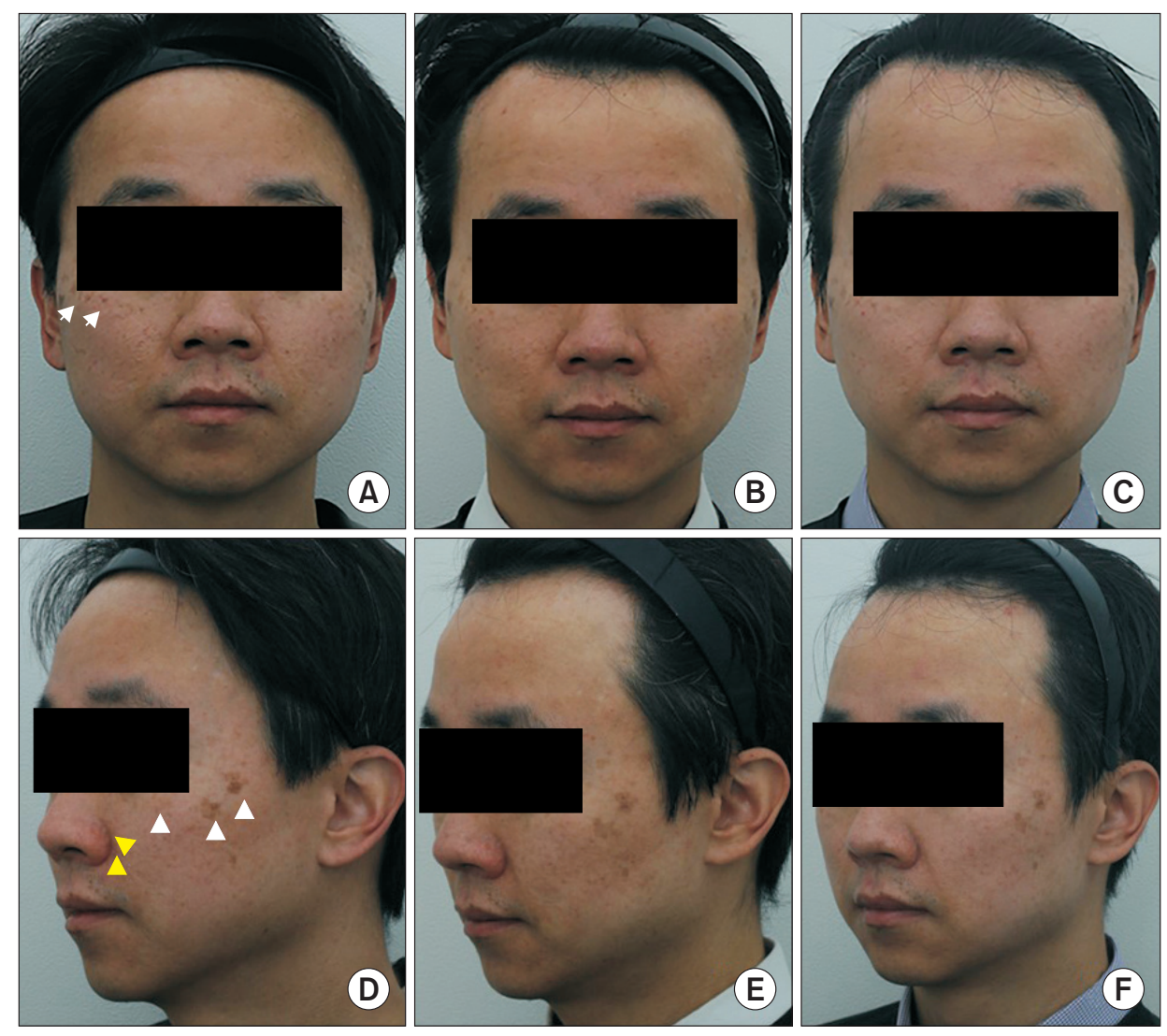

Fig. 3. A 43-year old man had very fragile skin in the sun and has suffered from multiple senile keratosis and erythema around the cheek area. (A, D) Before platelet-rich plasma (PRP) procedure there is severe erythema, multiple senile keratosis on the cheek. Telangiectasia is observed around the nasal alar area. (C, F) Postoperatively, erythema and senile keratosis much improved, and telangiectasia around the nasal alar area improved. Irregular skin texture due to sebum and moisture imbalance has been stabilized. White arrow: senile keratosis, yellow arrow: nasal alar area telangiectasia.

not fatal; however, it deteriorates the quality of a patient's life. Several treatment modalities may help control the inflammation and vasodilatation; however, there is currently no cure for rosacea. To solve the inflammatory response of the skin produced by immune dysfunction, the typical immunomodulation mechanism of PRP could be applied to rosacea. Casabona et al. [6] have reported that PRP is effective in the control of inflammation and skin regeneration in penile lichen sclerosus. More recently, PRP has been tested in patients with autoimmune diseases such as alopecia areata [7], rheumatoid arthritis, pemphigus vulgaris, and lichen planopilaris. PRP can attract peripheral blood monocytes in a dose-response manner, which leads to a change in the pro-inflammatory cytokine release profile [5].

We used the SmartPReP ${ }^{\circledR} 2 \mathrm{APC}_{+}{ }^{\mathrm{TM}}$ to prepare the PRP in the current procedures. In laboratory analysis using the Smart$\mathrm{PReP}^{\circledR} 2 \mathrm{APC}^{\mathrm{T}}{ }^{\mathrm{TM}}$, the platelet count was more than $1000,000 /$ $\mu \mathrm{l}$, which is roughly a 6 -fold greater concentrated platelet count than that in the peripheral blood [8]. Furthermore, the CD34 ${ }^{+}$ cell count was 13.9-31.1 cells/ $\mu \mathrm{l}$ and has a 2.1-4.7 fold more concentrated $\mathrm{CD}^{+} 4^{+}$cell count compared to peripheral blood mononuclear cells [8]. Zhong et al. [9] documented that bone marrow aspirate concentrate had $\mathrm{CD} 34^{+}$cells of $7.2 \% \pm 6.9 \%$,
PRP of $2.1 \% \pm 2.2 \%$, and peripheral blood of $0.48 \% \pm 0.6 \%$ via fluorescence-activated cell sorting analysis. Both human bone aspirate concentrates and PRP containing CD34 ${ }^{+}$cells had similar therapeutic effects on bone regeneration [9]. The CD34 hematopoietic stem cell is currently under investigation as a treatment for severe autoimmune diseases like alopecia universalis [6] and systemic sclerosis [10]. That study suggests the possibility of treating autoimmune diseases by the eradication of autoreactive cells.

Although there are numerous publications regarding the immunomodulatory mechanism of mesenchymal stem cells (MSCs), there are few articles on the treatment of immunerelated diseases with peripherally mobilized $\mathrm{CD} 34^{+}$hematopoietic stem cells. Preparing MSCs is not convenient as it requires invasive methods such as liposuction or bone marrow puncture. However, the peripheral blood can be easily collected through an intravenous draw.

We believe this is the first article to report the potential therapeutic effects of PRP enriched with $\mathrm{CD} 34^{+}$stem cells in the peripheral blood for the treatment of rosacea. In conclusion, PRP enriched with $\mathrm{CD} 34^{+}$stem cells in the peripheral blood may have a potential additive therapeutic effect on the treatment of 
rosacea. Long-term prospective follow-up studies are warranted to demonstrate a positive effect of PRP enriched with CD $34^{+}$ stem cells in the peripheral blood for the treatment of rosacea.

\section{Conflicts of interest}

The author has nothing to disclose.

\section{References}

1. Li Y, Xie H, Deng Z, Wang B, Tang Y, Zhao Z, et al. Tranexamic acid ameliorates rosacea symptoms through regulating immune response and angiogenesis. Int Immunopharmacol 2019;67:326-34.

2. Two AM, Hata TR, Nakatsuji T, Coda AB, Kotol PF, Wu W, et al. Reduction in serine protease activity correlates with improved rosacea severity in a small, randomized pilot study of a topical serine protease inhibitor. J Invest Dermatol 2014;134:1143-5.

3. van Zuuren EJ. Rosacea. N Engl J Med 2017;377:1754-64.

4. Kim MH, Byeon HS. Review for good platelet-rich plasma procedure in cosmetic dermatology and surgery. J Cosmet Med 2019;3:1-13.

5. Masoudi E, Ribas J, Kaushik G, Leijten J, Khademhosseini A.
Platelet-rich blood derivatives for stem cell-based tissue engineering and regeneration. Curr Stem Cell Rep 2016;2:33-42.

6. Casabona F, Gambelli I, Casabona F, Santi P, Santori G, Baldelli I. Autologous platelet-rich plasma (PRP) in chronic penile lichen sclerosus: the impact on tissue repair and patient quality of life. Int Urol Nephrol 2017;49:573-80.

7. Seifert B, Passweg JR, Heim D, Rovó A, Meyer-Monard S, Buechner S, et al. Complete remission of alopecia universalis after allogeneic hematopoietic stem cell transplantation. Blood 2005;105:426-7.

8. Kang JS, Zheng Z, Choi MJ, Lee SH, Kim DY, Cho SB. The effect of CD34+ cell-containing autologous platelet-rich plasma injection on pattern hair loss: a preliminary study. J Eur Acad Dermatol Venereol 2014;28:72-9.

9. Zhong W, Sumita Y, Ohba S, Kawasaki T, Nagai K, Ma G, et al. In vivo comparison of the bone regeneration capability of human bone marrow concentrates vs. platelet-rich plasma. PLoS One 2012;7:e40833.

10. Pawlak-Buś K, Schmidt W, Olejarz M, Czyż A, Komarnicki M, Leszczyński P. Autologous hematopoietic stem cell transplant for progressive diffuse systemic sclerosis: procedural success and clinical outcome in 5-year follow-up. Reumatologia 2019;57:50-4. 